University of Wollongong

Research Online

Australian Institute for Innovative Materials -

Papers

Australian Institute for Innovative Materials

$1-1-2015$

\title{
Nanocomposite hydrogels: Fracture toughness and energy dissipation mechanisms
}

Andrea Klein

University of Wollongong, andrea.klein@unileoben.ac.at

Philip G. Whitten

University of Wollongong, whitten@uow.edu.au

Katharina Resch

Montanuniversitat Leoben

Gerald Pinter

Montanuniversitat Leoben

Follow this and additional works at: https://ro.uow.edu.au/aiimpapers

Part of the Engineering Commons, and the Physical Sciences and Mathematics Commons

Research Online is the open access institutional repository for the University of Wollongong. For further information contact the UOW Library: research-pubs@uow.edu.au 


\title{
Nanocomposite hydrogels: Fracture toughness and energy dissipation mechanisms
}

\author{
Abstract \\ In this study, fracture toughness of nanocomposite hydrogels is quantified, and active mechanisms for \\ dissipation of energy of nanocomposite hydrogels are ascertained. Poly(N,N-dimethylacrylamide) \\ nanocomposite hydrogels are prepared by in situ free radical polymerization with the incorporation of \\ Laponite, a hectorite synthetic clay. Transmission electron microscopy proves exfoliation of clay platelets \\ that serve as multifunctional crosslinkers in the created physical network. Extraordinary high fracture \\ energies of up to $6800 \mathrm{~J} \mathrm{~m}-2$ are determined by the pure shear test approach, which shows that these \\ soft and stretchable hydrogels are insensitive to notches. In contrast to single- and double-network \\ hydrogels, dynamic mechanic analysis and stress relaxation experiments clarify that significant \\ viscoelastic dissipation occurs during deformation of nanocomposite hydrogels. Similar to double- \\ network hydrogels, crack tip blunting and plastic deformation also contribute to the observed massive \\ fracture energies.
}

\section{Keywords}

hydrogels, fracture, dissipation, nanocomposite, mechanisms, toughness, energy

\section{Disciplines}

Engineering | Physical Sciences and Mathematics

\section{Publication Details}

Klein, A., Whitten, P. G., Resch, K. \& Pinter, G. (2015). Nanocomposite hydrogels: Fracture toughness and energy dissipation mechanisms. Journal of Polymer Science Part B: Polymer Physics, 53 (24), 1763-1773. 


\title{
Nanocomposite Hydrogels: Fracture Toughness and Energy Dissipation Mechanisms
}

\author{
Andrea Klein, ${ }^{1,2}$ Philip G. Whitten, ${ }^{3}$ Katharina Resch, ${ }^{1}$ Gerald Pinter ${ }^{1}$ \\ ${ }^{1}$ Materials Science and Testing of Plastics, Department of Polymer Science, Montanuniversität Leoben, Otto Glöckel-Straße 2, \\ Leoben 8700, Austria \\ ${ }^{2}$ Faculty of Engineering, University of Wollongong, New South Wales 2522, Australia \\ ${ }^{3}$ ARC Centre of Excellence for Electromaterials Science, Intelligent Polymer Research Institute, AlIM Facility, Innovation Campus, \\ University of Wollongong, New South Wales 2522, Australia \\ Correspondence to: A. Klein (E-mail: andrea.klein@unileoben.ac.at)
}

Received 3 July 2015; accepted 31 August 2015; published online 8 October 2015

DOI: $10.1002 /$ polb.23912

ABSTRACT: In this study, fracture toughness of nanocomposite hydrogels is quantified, and active mechanisms for dissipation of energy of nanocomposite hydrogels are ascertained. Poly $(N, N$-dimethylacrylamide) nanocomposite hydrogels are prepared by in situ free radical polymerization with the incorporation of Laponite, a hectorite synthetic clay. Transmission electron microscopy proves exfoliation of clay platelets that serve as multifunctional crosslinkers in the created physical network. Extraordinary high fracture energies of up to 6800 $\mathrm{J} \mathrm{m}^{-2}$ are determined by the pure shear test approach, which shows that these soft and stretchable hydrogels are insensitive to notches. In contrast to single- and double-network hydro- gels, dynamic mechanic analysis and stress relaxation experiments clarify that significant viscoelastic dissipation occurs during deformation of nanocomposite hydrogels. Similar to double-network hydrogels, crack tip blunting and plastic deformation also contribute to the observed massive fracture energies. (c) 2015 Wiley Periodicals, Inc. J. Polym. Sci., Part B: Polym. Phys. 2015, 53, 1763-1773

KEYWORDS: crosslinking; fracture; fracture toughness; hydrogels; inorganic crosslinker; inorganic/organic network; nanocomposite hydrogels; networks
INTRODUCTION Hydrogels consist of polymer networks that contain a high fraction of water. By convention, the solvated polymer network chains are covalently linked by organic crosslinkers. ${ }^{1}$ Applications for hydrogels include wound dressings, ${ }^{2}$ incontinence pads, soft contact lenses, ${ }^{3}$ and water retention in agriculture. ${ }^{4}$ Many hydrogels are environmentally sensitive, that is, their water content, and hence size is dependent on temperature, humidity, and $\mathrm{pH}$ of their environment. ${ }^{5}$ This makes them highly attractive for stimuli-responsive biomedical applications, as their compliance is similar to mammal tissue. ${ }^{6}$ Potential future applications include artificial muscles ${ }^{7}$ and synthetic mammal tissue. However, to realize these applications, further development is necessary, as conventional hydrogels are inherently fragile. ${ }^{8}$ The polymer from which the hydrogel consists of is well above its glass transition with the solid being highly elastic. As there are no mechanisms for significant nonelastic dissipation of energy, conventional hydrogels are susceptible to minor cracks and flaws and exhibit brittle failure. ${ }^{9}$

There is much need to produce tough hydrogels, that is, hydrogels that are resistant to notches, so that their unique combina- tion of high compliance and high water content can be exploited. In the recent years, there have been many reports of novel synthesis routes that produce hydrogels that exhibit high tensile strain. However, as recently shown for a series of model hydrogels, ${ }^{10}$ a high tensile work of extension does not imply a high fracture toughness as predicted by the Lake-Thomas model. ${ }^{11}$

Double-network hydrogels are both the most characterized and best understood type of tough hydrogels. ${ }^{9}$ Double-network type hydrogels refer to hydrogels containing two interpenetrating networks with vastly different crosslink densities and where the network with the relatively large distance between crosslinks is in excess. The first generation of double-network hydrogels with a maximum toughness of $\sim 4000 \mathrm{~J} \mathrm{~m}^{-2}$ produce a massive increase in toughness by using network topology to increase the number of covalent bonds required for crack propagation. ${ }^{12,13}$ The second generation of double-network hydrogels that exhibit fracture energies in the vicinity of $9000 \mathrm{~J} \mathrm{~m}^{-2}$, as recently reported by Sun et al., ${ }^{14}$ used a similar network topology, but attained additional energy dissipation by exploitation of physical bonding within one network.

Additional Supporting Information may be found in the online version of this article.

(c) 2015 Wiley Periodicals, Inc. 
Of the different types of high work of extension hydrogels reported to date, nanocomposite hydrogels are the subject of the largest number of publications. ${ }^{15-17}$ Although strong nanocomposite hydrogels are best known because of their combination of ease of synthesis and their nonbrittle mechanical property, their toughness has not been quantified. The primary objective of this study is to quantify the tear toughness of high work of extension nanocomposite hydrogels. The secondary objective is to identify active mechanisms for dissipation of energy of nanocomposite hydrogels.

Nanocomposite hydrogels refer to highly stretchable polymer networks that contain both a high volume fraction of water and nanodimensioned inorganic particles. ${ }^{18}$ Various grades of Laponite, a synthetic hectorite clay, are the most reported type of particles used. It has been found that under specific conditions, the incorporation of Laponite as an inorganic crosslinker leads to nanocomposite hydrogels that exhibit extraordinary characteristics, including outstanding transparency, rapid swelling and deswelling properties, high tensile strength combined with high elongation at break, and high compliance. ${ }^{1}$ Network-forming physical bonds form crosslinks between organic linear polymer chains and inorganic clay discs. Nanocomposite hydrogels are known to exhibit a large area under their tensile stress-strain curves, ${ }^{19}$ indicating a high work of extension. However, the tearing toughness of nanocomposite hydrogels without chemical crosslinks has not yet been reported.

The hydrogels reported in this study consist of $\operatorname{poly}(\mathrm{N}, \mathrm{N}$ dimethylacrylamide) (PDMAA) as the polymer and Laponite XLS as the inorganic crosslinker. For Laponite-type hydrogels, poly( $N$-isopropylacrylamide) (PNIPA) has the highest number of citations. However, PNIPA exhibits a lower critical solution temperature of $\sim 32{ }^{\circ} \mathrm{C}$, which can complicate synthesis, analysis, and interpretation of results. Similar to PNIPA, PDMAA was reported to produce excellent nanocomposite hydrogels with Laponite XLG as an inorganic crosslinker. ${ }^{20}$ In comparison with a PDMAA-XLG hydrogel with a clay content of 4.5 wt \% ("NC7," using the nomenclature of Haraguchi et al.), a representative PNIPA-XLG hydrogel with the same clay and polymer content (by weight) exhibits $\sim 0.5 \times$ the elastic modulus, $0.8 \times$ the breaking strength, and $1.1 \times$ the elongation at break. ${ }^{15}$ Hence, when synthesized appropriately, PDMAA-XLG hydrogels are comparable with PNIPA-XLG at similar polymer and clay contents.

The type of Laponite clay used in the synthesis of nanocomposite hydrogel is also important. Laponite XLS refers to a specific type of tetrasodium pyrophosphate-modified XLG. Laponite XLG is known to rapidly form gels when mixed with water at concentrations of 3 wt \% or greater. Hence, to synthesize hydrogels with high clay content, detailed process-sensitive multistep procedures are necessary. In contrast, dispersed Laponite XLS will temporarily remain a liquid at concentrations as high as 11 wt $\%$ for at least $12 \mathrm{~h}$. Hence, the use of Laponite XLS instead of Laponite XLG simplifies the hydrogel synthesis when concentrations higher than 3 wt \% are needed. When Laponite XLS is dispersed in water, pyrophosphate anions associate with the positively charged Laponite crystal edge leading to repulsion. However, the pyrophosphate anions are not stable in water and over time degrade forming phosphate anions. The phosphate anions do not associate with the Laponite edge, and when the Laponite XLS concentration is sufficiently high, a gel will form after a period of time similar to Laponite XLG. Any mechanical properties associated with clay-clay interactions are likely to be reduced when the XLS form of Laponite is used in comparison with the XLG form. ${ }^{21}$

\section{EXPERIMENTAL}

Nanocomposite and organic hydrogels were prepared using DMAA from Sigma Aldrich (St. Louis, Missouri) as monomer and clay of type Laponite XLS ${ }^{\circledR}$, a synthetic hectorite (Rockwood Additives, Princeton, NJ) as inorganic crosslinker. $N, N^{\prime}$ Methylenebis(acrylamide) (BIS; Sigma Aldrich) was used as organic crosslinker. Potassium persulfate from Sigma Aldrich was used as initiator. $N, N, N^{\prime}, N^{\prime}$-Tetramethylethylenediamine from Sigma-Aldrich was used as accelerator. Water used for sample preparation was obtained from an Elix 3 Water Purification System, EMD Millipore Corporation, Billerica, MA (resistivity $>5-15 \mathrm{M} \Omega \mathrm{cm}$ ).

Preparation of nanocomposite hydrogels was conducted as follows. Clay was added to $19.5 \mathrm{~mL}$ of precooled pure water, forming an opaque solution. The mixture was continuously stirred in an ice bath with a magnetic stirrer at $\geq 300 \mathrm{rpm}$. Then, $4 \mathrm{~mL}$ of monomer was added. When the mixture turned transparent indicating the dispersion of clay (after $\sim 1 \mathrm{~h}$ ), $30 \mu \mathrm{L}$ of accelerator and $2 \mathrm{~mL}$ of an aqueous solution containing initiator were added. Thereafter, the solution was poured into a polypropylene (PP) bag with a funnel. The bag was subsequently squashed between two precooled glass slides and fixed with clips. Plastic spacers were used to control the thickness of the samples ( $2 \mathrm{~mm}$ ). Next, oxygen was removed from the sample by placing it in a chamber where gas was removed by a vacuum pump followed by purging the chamber with nitrogen gas. The vacuuming/purging cycle was repeated three times. In situ free radical polymerization was allowed to proceed for $\sim 55 \mathrm{~h}$ at ambient temperature. After polymerization, all samples were stored in airtight containers to avoid drying out. The PP bags were peeled off from the hydrogel surfaces immediately before testing. Preparation of organic hydrogels follows the same procedure where clay was substituted by the organic crosslinker. The compositions of the samples are shown in Table 1. The nomenclature used to identify each hydrogel (e.g., NC7) was developed by Haraguchi et al. ${ }^{15}$

X-ray diffractometry (XRD) with a GBC instrument was conducted on freeze-dried and ground samples to study clay exfoliation. Scans are performed between $1.8^{\circ}$ and $10^{\circ}$ at $0.5^{\circ} \min ^{-1}$ with a step size of $0.02^{\circ}$ using $\mathrm{Cu} \mathrm{K} \alpha$ radiation with graphite monochromators. Samples are dispersed on low background-oriented quartz substrates. The scans are conducted at $30 \mathrm{~mA}$ and $40 \mathrm{kV}$. 
TABLE 1 Compositions of the Reagents Used to Synthesize the Hydrogels

\begin{tabular}{lllll}
\hline & \multicolumn{4}{c}{ Composition } \\
\cline { 2 - 5 } Hydrogel & Clay $(\mathrm{g})$ & BIS $(\mathrm{g})$ & DMAA $(\mathrm{mL})$ & Water $(\mathrm{mL})$ \\
NC7 & 1.15 & - & 4.00 & 21.5 \\
NC9 & 1.48 & - & 4.00 & 21.5 \\
NC11 & 1.80 & - & 4.00 & 21.5 \\
NC13 & 2.13 & - & 4.00 & 21.5 \\
OR & - & 0.298 & 4.00 & 21.5
\end{tabular}

Transmission electron microscopy (TEM) was conducted on freeze-dried samples, sectioned using a Leica Ultra-Cut 7 microtome (Leica Microsystems, Wetzlar, D) with glass knives to yield ultrathin films of about 70-90 nm thickness using a JEM 2011 TEM (Jeol, JP) as to verify clay exfoliation and distribution of clay.

All mechanical testing was conducted with a universal testing machine (Instron 5566; Instron). For tensile, step cycle, and relaxation tests, specimens were cut to a dumbbell shape according to ISO 27:2011 (Type 2), and the axial strain was recorded by a video extensometer (Advanced Video Extensometer 2663-821; Instron).

Tensile testing was performed at a constant crosshead speed of $100 \mathrm{~mm} \mathrm{~min}^{-1}$ to obtain stress-strain curves. Additionally, the modulus is evaluated from a secant between 0 and $5 \%$ of strain. For strains larger than 350\%, a mounted JVC GZ HM445BAA camcorder was used to determine axial strain.

Step cycle tests, used to determine the ratio between elastic and plastic deformation, were performed at a strain rate of $0.5 \% \mathrm{~s}^{-1.22}$ Specimens were elongated to a strain $\varepsilon_{x}$ $(25 \times x \%)$, where $x$ is the cycle number, followed by unloading to $0 \mathrm{kPa}$ stress. Fourteen cycles were conducted attaining a maximum strain of $350 \%$. Specimens were coated with silicone oil after mounting to avoid dehydration. Resulting true stress-true strain curves are referred to for evaluation.
For stress relaxation experiments, samples were stretched to $100 \%$ at a constant crosshead speed of $1.67 \mathrm{~mm} \mathrm{~s}^{-1}$. The strain was held constant for $\sim 1500 \mathrm{~s}$, whereas the tensile stress was recorded as a function of time. Evaluation of stress relaxation data uses a two-element Kelvin model as stated in the following formula:

$$
\sigma(t)=\sigma_{\infty}+\sigma_{1} e^{\left(\frac{-t}{\tau_{1}}\right)}+\sigma_{2} e^{\left(\frac{-t}{\tau_{2}}\right)}
$$

where $\sigma_{\infty}$ is the tensile stress at infinite time, $\sigma_{1}$ and $\sigma_{2}$ represent $E_{1} \times \varepsilon_{0}$ and $E_{2} \times \varepsilon_{0}$, respectively, $\tau_{1}$ and $\tau_{2}$ are the relaxation times for short and long relaxation processes, respectively. $E \infty, E_{1}$, and $E_{2}$ represent relaxation moduli, and $\varepsilon_{0}$ represents the initial stretch.

Fracture toughness was determined by a single notch tear test with a pure shear geometry ${ }^{14,23}$ at a crosshead speed of $10 \mathrm{~mm} \mathrm{~min}^{-1}$. Specimen dimensions were $5 \mathrm{~mm}$ long $\times$ $70 \mathrm{~mm}$ wide. A single notch of $30 \mathrm{~mm}$ in length was made via scissors and sharpened with a razor blade after mounting. Both scissors and razor blade were coated with silicone oil to prevent the material from adherence. Inherent to the pure shear geometry, the sample is much wider than it is long, and hence, gripping the compliant samples without squeezing or slipping is difficult. For the nanocomposite hydrogels described here, the approach described by Sun et al. ${ }^{14}$ where samples are glued to spaced plastic microscope slides was not satisfactory as the samples de-adhered from the grips during testing. Consequently, a 3D printer was used to fabricate custom grips containing the following features (see Figure 1 and Supporting Information): raised pillars and holes were added to the grip surface to increase contact area, the holes also enabled the deposition of cyanoacrylate glue (Zap-A-GAP CA+; Pacer Technologies, CA) after clamping which prevented the spreading of glue over the sample surface; spacers were used to control the grip separation at $2 \mathrm{~mm}$; locating pins were used to control the grip spacing; and the grips contained a thin frame that was cut after mounting in the testing machine. Comparison of grip displacement with the displacement of dust particles on the hydrogels surface shows that the hydrogel is not being

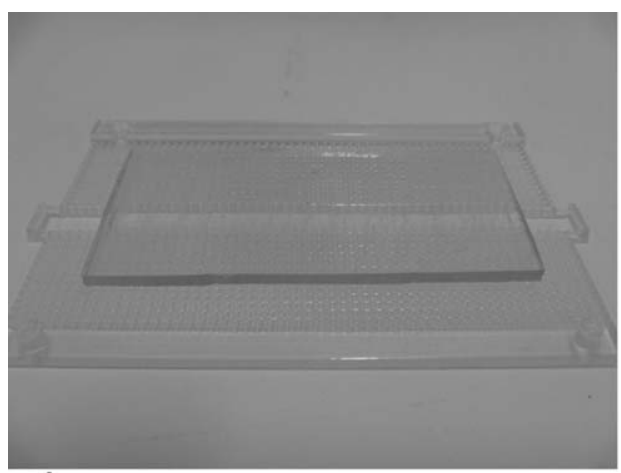

a)

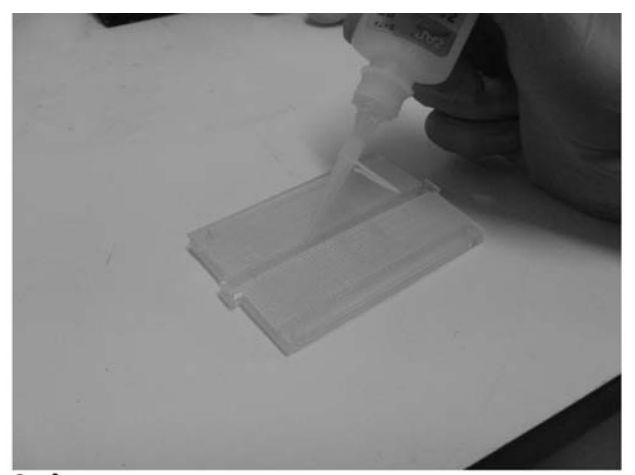

b)

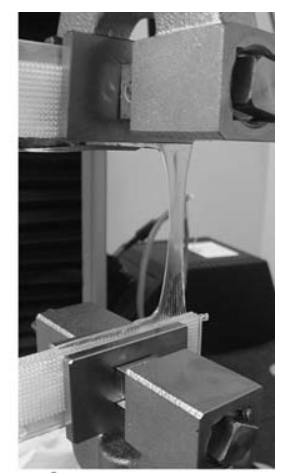

c)

FIGURE 1 Gel samples were placed on top of a set of grips (a) and then were clamped by placing the alternate grip surface on top of the gel and applying glue (b). A notched sample during a tearing toughness test (c). 

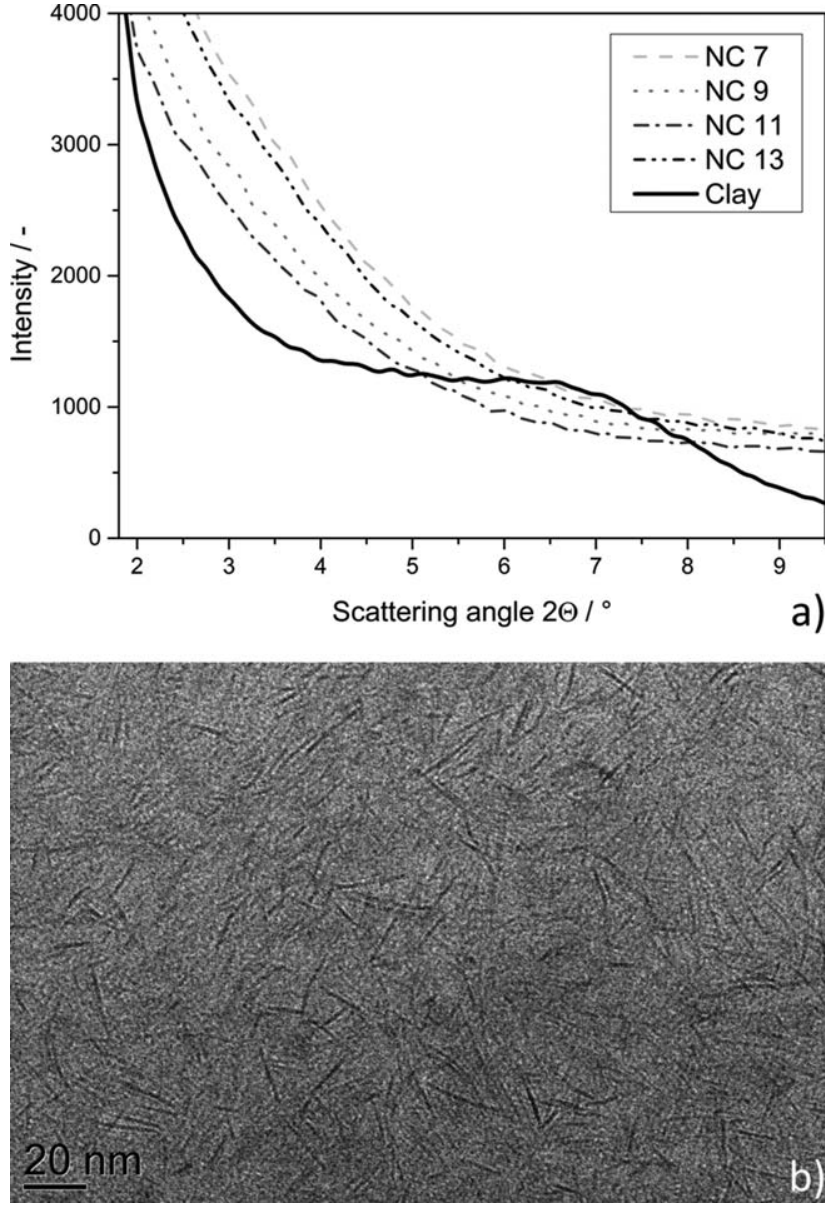

FIGURE 2 (a) XRD spectra of nanocomposite hydrogel samples and pure clay. (b) TEM image of sample NC9 clay particles of longish shape.

displaced from the clamps during testing (see Figure 3 and Supporting Information). The fracture toughness was evaluated from the force-displacement curves according to Sun et al. $^{14}$ and is described briefly in the Supporting Information.

Dynamic mechanic analysis was performed on a Solids analyzer DMA (RSA-G2; TA Instruments). Testing was conducted at $\pm 1 \%$ shear strain over the frequency range from 0.01 to $10 \mathrm{~Hz}$ at ambient temperature using shear sandwich clamps. Specimens consist of pairs of rectangular shaped test pieces $(13 \mathrm{~mm} \times 16 \mathrm{~mm})$.

\section{RESULTS AND DISCUSSION}

\section{Morphological Analysis}

Morphological analysis including XRD as well as transmission electron microscopy were conducted as to verify sufficient sample preparation in terms of ensuring sufficient polymerization conditions and uniform distribution of clay platelets. Figure 2 (left) shows XRD spectra of the samples NC7, NC9, NC11, and NC13 as well as of pure clay as received. The clay exhibits a diffraction band with a center

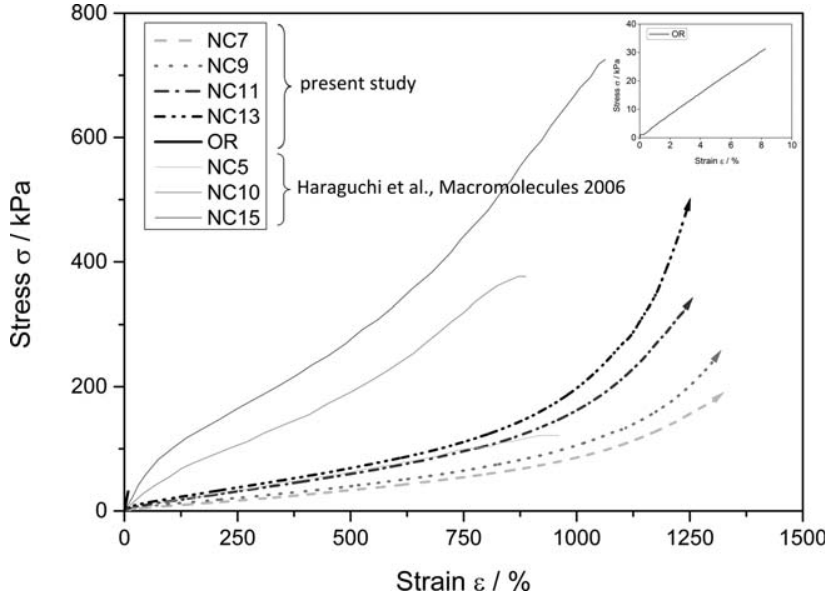

FIGURE 3 Simple extension stress-strain curves for PDMAANC samples NC7, NC9, NC11, NC13, and OR for tensile strains within the crosshead range of motion. In addition, stress-strain data extracted from Haraguchi et al. ${ }^{19}$ for PNIPA-NC hydrogels are plotted. Reprinted (adapted) with permission from Haraguchi and Li, Macromolecules, 2006, 39 (5), 1898-1905. Copyright 2006 American Chemical Society. The inset shows sample OR in more detail.

at about $6.5^{\circ}$. According to the Bragg's Law, the basal spacing of the clay is about $1.4 \mathrm{~nm}$.

All nanocomposite hydrogel samples exhibit XRD spectra of the same form that is different to that for the neat clay. The different levels of scattering intensity observed for the dried hydrogel samples might be related to the grade of coarseness, which is gained by grinding the samples to powder. No diffraction bands are present in the measured scattering angle range. Thus, it is concluded that clay in the hydrogel samples does not layer regularly but instead exfoliates and disperses randomly.

TEM analysis of nanocomposite hydrogels exhibits uniformly dispersed straight linear particles with a length of $\sim 20$ to $30 \mathrm{~nm}$ and a thickness of $\sim 1$ to $1.5 \mathrm{~nm}$. These particles display exfoliated clay sheets. It is assumed that the drying process necessary for TEM analysis is unable to exfoliate or further separate adjacent clay particles. It is concluded that the clay in the nanocomposite hydrogels reported here is fully exfoliated and dispersed uniformly. Figure 2 (right) displays an exemplary TEM image of sample NC9.

The morphological analysis shows that the clay is exfoliated and homogenously distributed within the nanocomposite hydrogels, a common feature of nanocomposite hydrogels exhibiting high work of extension.,15,20

\section{Mechanical Properties \\ Simple Extension Stress-Strain}

Simple extension stress-strain curves of organic hydrogels and nanocomposite hydrogels are plotted in Figure 3. The representative stress-strain curves extracted from the seminal work of Haraguchi et al. on poly- $N$-isopropyl 


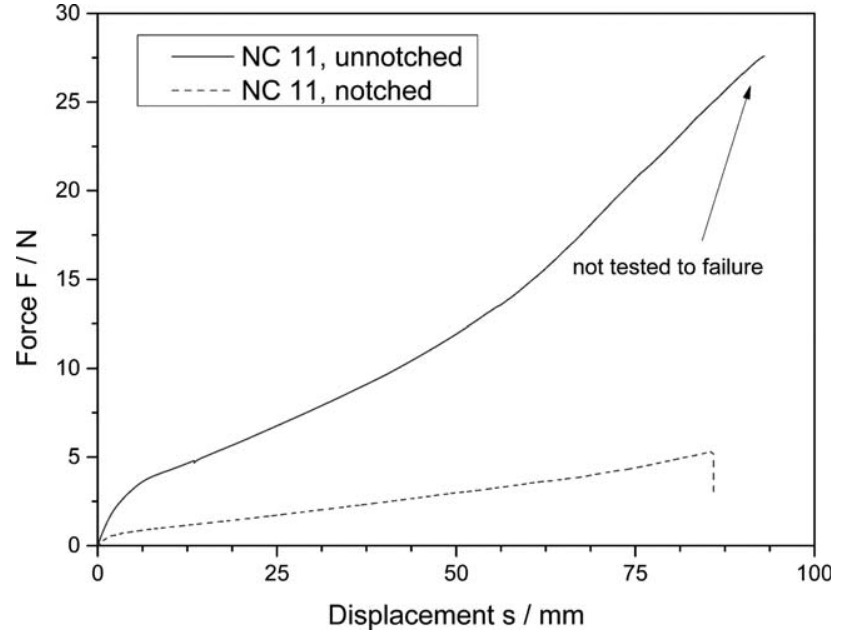

FIGURE 4 Pure shear force-displacement curves (solid line: unnotched specimen; dashed line: notched specimen) for sample NC11. Testing of unnotched specimen was deliberately stopped.

acrylamide nanocomposite hydrogels with high clay contents are also included in Figure $3 .^{19}$ The organic hydrogel OR shows brittle characteristics, with a strain-at-break of $\sim 10 \%$, stress-at-break of about $30 \mathrm{kPa}$, and a Young's modulus of $378 \mathrm{kPa}$. In contrast, the nanocomposite hydrogels exhibit massive extendibility, high work of extensions, and did not break at tensile strains $\leq 1250 \%$, which corresponds to the testing machine's maximum crosshead distance of travel. An increase of clay content results in higher stress levels at equivalent strain. Furthermore, an increase of clay content leads to monotonically increasing values from a Young's modulus of $31 \mathrm{kPa}$ for sample NC7 up to $99 \mathrm{kPa}$ for sample NC13 (see Fig. 4 and Supporting Information). Note that at the concentrations of Laponite used here, a neat Laponite suspension is a thixotropic material, which exhibits a yield stress if left at rest. Hence, the Young's modulus for nanocomposite hydrogels is the result of rubber elasticity of the polymer chains as well as clay-clay interactions.

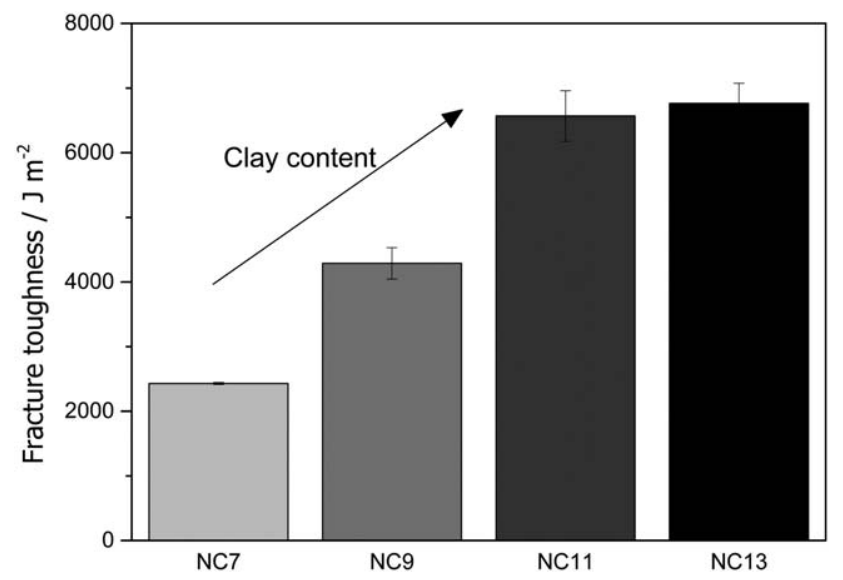

FIGURE 5 Fracture toughness in dependence of clay content for samples NC7, NC9, NC11, and NC13 including standard deviation.
The tensile properties of the nanocomposite gels reported here are similar to those reported by others. For example, Haraguchi et al. ${ }^{19}$ prepared hydrogels with similar clay content, but, with PNIPA and Laponite XLG that exhibited two to three times of the stress detected for hydrogels in the present investigations at equivalent strains, but, a lower elongation at break. The same group ${ }^{20}$ also reported a DMAALaponite XLG nanocomposite hydrogel with a clay content consistent with NC7 that exhibited a Young's modulus of 16 $\mathrm{kPa}$, a breaking strength of $256 \mathrm{kPa}$, and an elongation at break of $1319 \%$, which are similar to those reported here.

It has been shown that clay-clay interactions are responsible for a major fraction of the Young's modulus. ${ }^{24,25}$ To estimate the contribution of polymer chain elasticity to the Young's modulus, the tensile test data were applied to a Mooney plot. $^{26,27}$ The tangent of the Mooney plot is attributed to polymer chain elasticity. Hence, it was estimated that the polymers contribution to Young's modulus ranges from $11 \mathrm{kPa}$ for NC7 up to $26 \mathrm{kPa}$ for NC13, that is, $\sim 1 / 3$ of the Young's modulus at low strains is attributed to polymer elasticity.

Rubber elasticity theory applied to polymer gels predicts that the Young's modulus is proportional to the number of polymer chains per unit volume. The Young's moduli for the nanocomposite gels are an order of magnitude lower than that of the organic hydrogel, indicating that the number of chains per unit volume in the nanocomposite gels is at least an order of magnitude lower than that for the organic gel.

The ratio of tensile stress to modulus $\left(\sigma_{\mathrm{b}}: E\right)$ is useful for predicting the possibility of elastic crack tip blunting. For the organic hydrogel, $\mathrm{OR}, \sigma_{\mathrm{b}}: E$, the elastic modulus is 0.08 hence no elastic crack tip blunting will occur. ${ }^{28}$ For the nanocomposite gels, the tensile stress was not determined. However, at a strain of $350 \%$, the $\sigma_{350 \%}: E$ ranges from 0.49 to 0.74 , but would be $>1$ if one considered only the polymer chain elasticity contribution to modulus. Hence, for the nanocomposite gels, significant elastic crack blunting will occur.

\section{Tearing Toughness}

Figure 4 displays representative force-displacement curves for both a notched and an unnotched NC11 specimen using the pure shear test approach. For the notched samples, displacement-at-break is between 70 and $112 \mathrm{~mm}$ (corresponding to tensile strains of 1400 and 2240\%). Testing of unnotched samples was stopped (end of full line in Fig. 4 when the elongation exceeded the displacement-at-break of corresponding notched samples).

Evaluation regarding fracture toughness yields a fracture toughness between 2400 and $6800 \mathrm{~J} \mathrm{~m}^{-2}$ depending on clay content as displayed in the bar diagram (Figure 5) including standard deviations of the average. An increase of clay content leads to higher fracture toughness. This is in agreement with an increase in work of extension observed during tensile testing measurements. Large increases in fracture toughness were observed as the clay concentration was increased from 7 to $11 \mathrm{~mol} \mathrm{~L}^{-1}$ water. For this clay content, fracture toughness of 


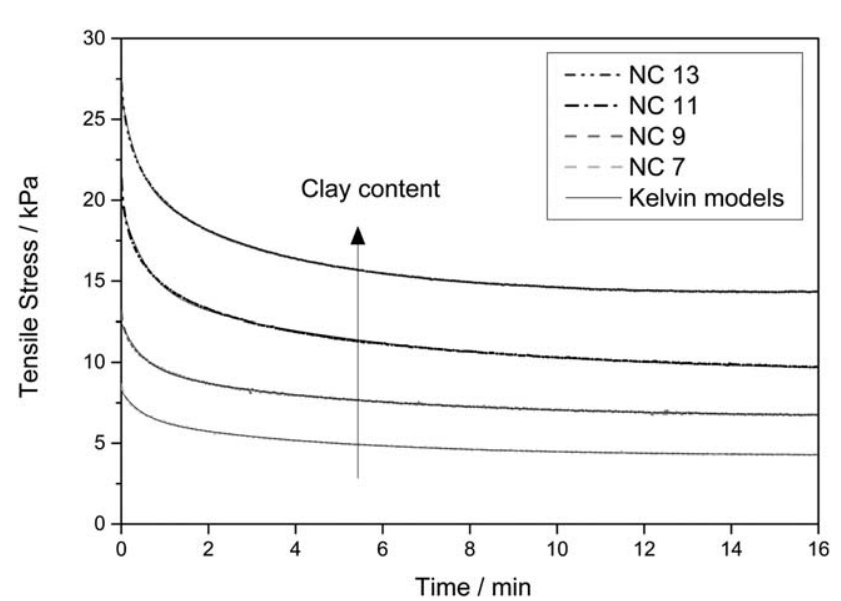

FIGURE 6 Stress relaxation curves for samples NC7, NC9, NC11, and NC13 elongated to $100 \%$ strain (solid lines: experimental observation; dashed lines: corresponding fitted Kelvin models).

$6600 \mathrm{~J} \mathrm{~m}^{-2}$ is yielded. The determined fracture toughness of NC11 and NC13 are equivalent as they are within experimental error. When increasing the clay content from 11 to 13 mol L ${ }^{-1}$ water, there was a large increase in fracture force for notches of equivalent size; however, there was also a large decrease in displacement at fracture leading to equivalent fracture toughness. Hence, it is predicted that a further increase in clay content beyond $13 \mathrm{~mol} \mathrm{~L}^{-1}$ would not result in significantly higher fracture toughness than observed for sample NC13 because of an anticipated reduction in displacement at break. No fracture toughness testing was conducted on sample OR because of its inherent fragility.

It is worth noting that visual observations during experiments reveal that blunting of the cracks starts with the onset of grip displacement, which is expected because of the high ratio of tensile stress to elastic modulus. Blunting is an important toughening mechanism, ${ }^{29}$ which contributes to the high fracture toughness by reducing the stress concentration at the crack tip. The nanocomposite hydrogel samples were not susceptible to notches. One consequence of this insen-

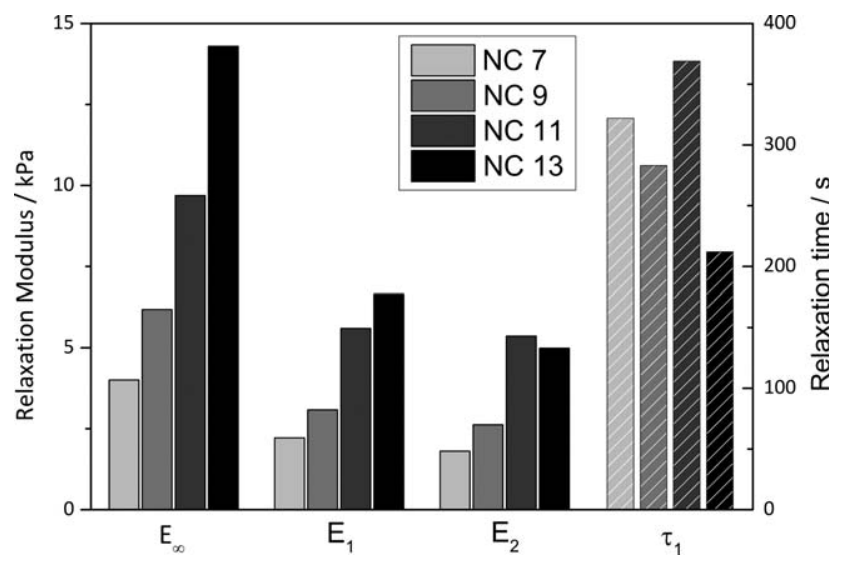

FIGURE 7 Relaxation moduli and relaxation time 1 for samples NC7, NC9, NC11, and NC13. sitivity to notches is the large difference in force levels between notched and unnotched samples. As notching samples solely cause a reduction of cross section, constant stress (quotient of force and area) results in a higher force for unnotched samples when compared with notched samples.

A comparison with reported fracture toughness for other types of gels reveals that fracture toughness of nanocomposite hydrogel samples considered in this study are very high. They are substantially tougher than the less compliant first generation of double-network hydrogels that exhibited a maximum toughness of $\sim 4000 \mathrm{~J} \mathrm{~m}^{-2}$. They are less tough than the extraordinary tough double-network gels synthesized by Sun et al. ${ }^{14}$ that exhibited a maximum toughness of $8700 \mathrm{~J} \mathrm{~m}^{-2}$. The gels prepared by Sun et al. consist of an ionically crosslinked alginate interpenetrating covalently crosslinked polyacrylamide. $^{14}$

\section{Viscoelastic Energy Dissipation}

Viscoelasticity is characterized at long-time scales by stress relaxation experiments and at short-time scales by dynamic mechanic analysis.

Figure 6 displays stress relaxation curves of nanocomposite hydrogel samples following stretching to $100 \%$ strain. After $\sim 300$ s, stress reaches a plateau as known for crosslinked polymers. The observed stress relaxation plots could not be described by a single-element Kelvin model. Fitting of twoelement Kelvin models yield correlation coefficients higher than $0.995 . E_{\infty}$ represents the long-term Modulus after the sample has relaxed, whereas $E_{1}$ and $E_{2}$ describe the timedependent fraction of the stiffness. The sum of $\left(E_{1}+E_{2}\right.$ $\left.+E_{\infty}\right)$ represents the Modulus present instantly after applying an external load and is less than the Young's modulus observed for simple extension tests as the applied strain is in the region where stress is no longer proportional to strain. $E_{\infty}$ and $E_{1}$ increase with increasing clay content. $E_{2}$ also increases with increasing clay content; however, samples NC11 and NC13 exhibit similar values. Figure 7 displays a bar chart containing relaxation moduli.

Both $\tau_{1}$ and $\tau_{2}$ show no correlation to clay content. From stress relaxation experiments, it is concluded that substantial viscoelastic dissipation occurs when nanocomposite hydrogels are held at constant length, with a maximum dissipation coinciding with a relaxation time $\tau_{1}$ of $290 \mathrm{~s}$. Relaxation time $\tau_{1}$ is of the same order as the duration of notched pure shear tests $(\sim 600 \mathrm{~s})$. This implies that viscoelastic dissipation energy associated with stress relaxation significantly contributes to the observed high fracture energies. Both $\tau_{2}$ (average of $14 \mathrm{~s}$ ) and $E_{2}$ are likely to be underestimated as $\tau_{2}$ is less than the loading time, consequently $\tau_{2}$ and $E_{2}$ are likely to exhibit significant error.

In general, the relaxation effect is due to time-dependent processes. The chains cannot follow the deformation simultaneously. Polymer chain translation and clay platelet orientation are required to allow for a lower stress level in the strained state likely to coincide with stress relaxation. 


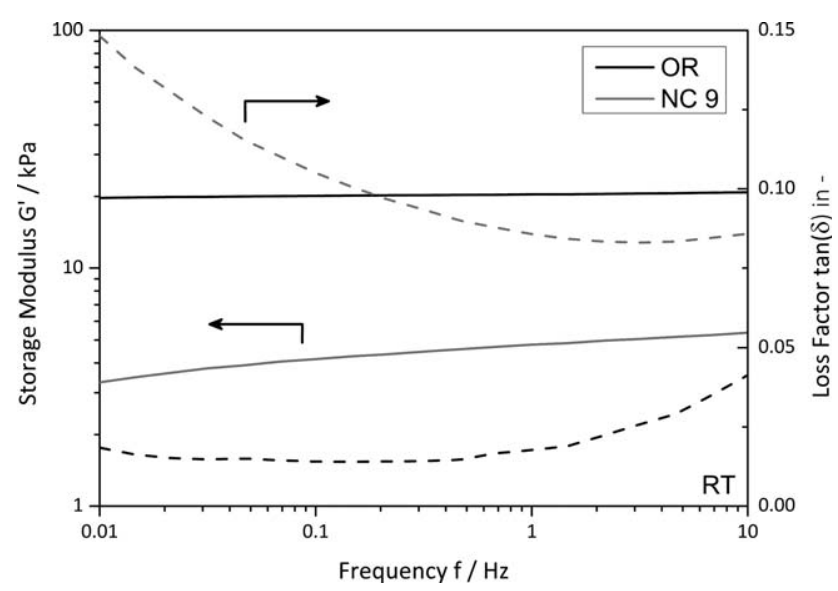

FIGURE 8 Storage shear modulus (solid lines) and loss factor (dashed lines) as a function of frequency for samples NC9 and OR.

Specifically, the rupture and formation of clay-clay and polymer-clay bonds control the rate of chain translation and platelet orientation. As the lifetime of these bonds is a function of force, ${ }^{30}$ it is anticipated that the relaxation times would also be a function of applied strain. In contrast, OR is assumed to perform in an elastic manner ${ }^{18}$ and should not exhibit a relaxation effect.

Dynamic mechanic analysis (DMA) showed that significant dissipation of energy occurs at time scales that are short relative to the tear test. Figure 8 shows the storage modulus and factor as a function of frequency for OR and NC9. As expected, the storage modulus of the organic gel is almost an order of magnitude higher than that of the nanocomposite gels. In addition, as expected, the dissipation of energy represented as the loss factor was much larger for nanocomposite gels relative to organic gels. The form and magnitude of the DMA results for the OR gel are consistent with those reported by Sun et al. ${ }^{14}$ for a conventional polyacrylamide gel. Specifically, the storage modulus is almost independent of oscillation frequency, whereas there is a small increase in the loss factor as the oscillation frequency is increased from 0.01 to $10 \mathrm{~Hz}$. In contrast, for the nanocomposite gels, there was a marked increase in storage modulus that coincided with a substantial decrease in loss factor as the oscillation frequency was increased from 0.01 to $10 \mathrm{~Hz}$. The opposite correlation between both storage modulus and loss factor against oscillation frequency was reported by Sun et al. for alginate-type physically bonded hydrogels. ${ }^{14}$

The mechanism for viscoelastic energy dissipation observed by the DMA in the nanocomposite gels is likely to be timedependent clay-clay or polymer-clay interactions. The energy dissipation cannot be attributed to polymer-polymer interactions, entanglements, segmental, or pendent group mobility as OR consisted of the same polymer. It is well known that there is a correlation between force and lifetime of noncovalent molecular bonds, ${ }^{30}$ which can explain both an increase in modulus and a decrease in loss factor with an increase in oscillation frequency. The lifetime of the interactions responsible for the viscoelastic loss at the applied displacement are much longer than $100 \mathrm{~s}$ (corresponding to an oscillation frequency of $0.01 \mathrm{~Hz}$ ). This is consistent with the observation that the nanocomposite hydrogel is a solid in the situation that the same interactions are responsible for physical crosslinks. It is pertinent to note that the DMA does not display a maximum that is consistent with the relaxation time $\tau_{2}$ observed in stress relaxation experiments. This would suggest that the relaxation time $\tau_{2}$ is associated with a mechanism that requires a deformation greater than $1 \%$, for example, translation of polymer chains.

For the nanocomposite gels, the dynamic storage modulus increases monotonically with clay content, whereas at a frequency of $10 \mathrm{~Hz}$, the loss factor decreases monotonically with clay content, with increasing frequency as well as with increasing clay content (Figure 9). For instance, the modulus at $1 \mathrm{~Hz}$ is $3.6 \mathrm{kPa}$ for sample NC7 and $7.8 \mathrm{kPa}$ for sample NC13. Clay-clay interactions are mainly responsible for the DMA-determined moduli due to the small shear strains. ${ }^{24,25}$ Hence, it is expected that DMA storage modulus should increase with clay content as there is a greater density of clay-clay interactions.

For nanocomposite hydrogel samples, the loss factor lies between 0.17 and 0.05 , which are comparably small values. Hence, the samples are essentially elastic for deformation of $1 \%$ shear strain. As the loss factor, representing viscoelastic dissipation, can be considered as the degree of a material's toughness, this result indicates that the high fracture toughness of nanocomposite hydrogels in consideration is not attributed to loss from small deformations. ${ }^{31}$

\section{Plastic Dissipation}

To analyze the extent of plastic dissipation during deformation, step cycle testing is performed on nanocomposite hydrogels. Note that the deformation referred here to as "plastic" is not only strictly plastic but also contains a slow elastic component. Here, the purpose is to isolate what

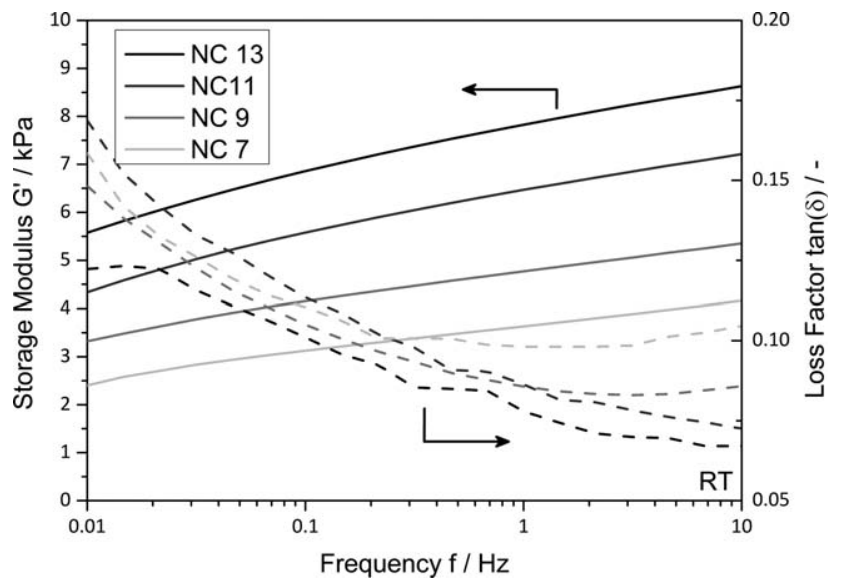

FIGURE 9 Storage shear modulus (solid lines) and loss factor (dashed lines) as a function of frequency for samples NC7, NC9, NC11, and NC13. 


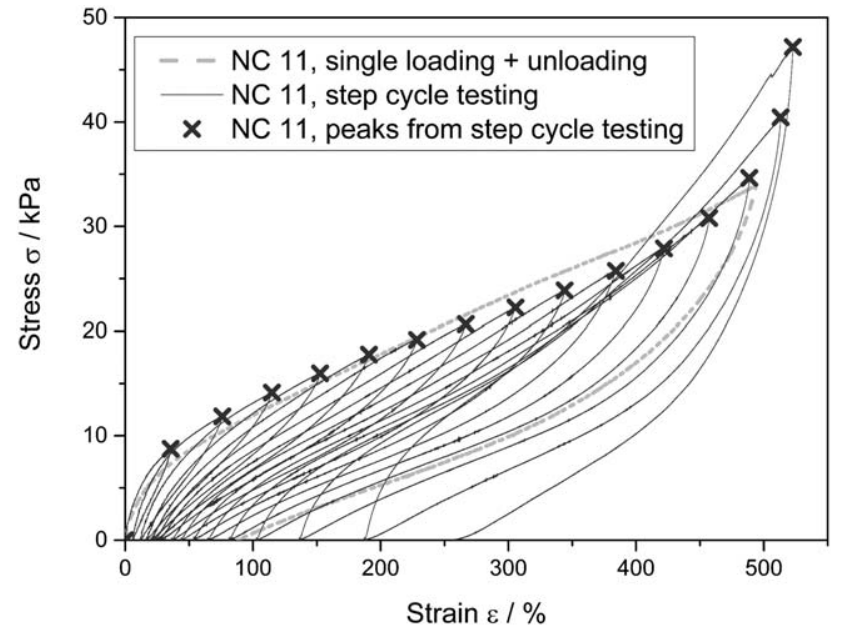

FIGURE 10 Step cycle testing versus single load testing for representative sample NC11. Crosses indicate reversal points from loading to unloading in step cycle testing.

fraction of the work of extension would not contribute to a propagating tear; consequently, the slow elastic component should be combined with viscous plastic component.

Figure 10 displays an exemplary stress-strain curve as obtained from step cycle testing, plotted as solid line. Crosses highlight peaks which correspond to the reversal point from loading to unloading. In Figure 10(a), the constant strain rate simple extension test conducted on the same type of sample is shown for comparison. For all of the nanocomposite gels, at strains ranging from 425 to $500 \%$, the maximum stress that coincides with the maximum cycle strain becomes substantially larger than that of a single-cycle continuous extension. This maximum stress that is beyond that observed for a single-cycle continuous extension corresponds to an increase in the tangent modulus is due to a cycle-induced strain hardening. Cycle-induced strain hardening of nanocomposite gels has been described elsewhere ${ }^{19}$ and corresponds to a cycleinduced orientation of the clay platelets. Two consequences of the cycle-induced strain hardening are for the purpose of evaluating the energy dissipated via slow elastic and plastic deformation, the step cycle test is only valid for strains less than the onset of cycle-induced strain hardening, and the tearing toughness of nanocomposite gels is likely to be cycle-dependent.

The ratios between elastic and plastic strains against the total strain as a function of true strain are presented in Figure 11. Supporting Information Figure S5 shows schematically how the elastic and plastic strains were determined. The ratio between elastic and plastic strain is nearly constant up to a total true strain of about 1.2 to 1.5 . The total strain consists of an $80 \% \pm 5 \%$ elastic fraction and a $20 \% \pm 5 \%$ plastic fraction. However, the plastic fraction of work of extension is much larger than the plastic fraction of the total strain. Hence, the nanocomposite hydrogels exhibit substantial hysteresis when exposed to single tensile cycle. It is clear that a major fraction of the work of extension would not be released rapidly if a tear was propagating, which is a major reason for the high-observed tear toughness. It must be noted that for higher strains, the extent of plastic strain will be overestimated by up to $30 \%$ in comparison with a sample that is left for a day or more without load between cycles. ${ }^{19}$ However, it is pertinent to note that such a difference would increase the hysteresis reported and result in a higher toughness if a tear test was conducted at substantially slower rates. Condensed, step cycle testing reveals that plastic dissipation substantially contributes to the high fracture energies of nanocomposite hydrogels.

\section{Why Do Nanocomposite Gels Exhibit High Toughness?}

The four key mechanisms to achieve high toughness are a low ratio of elastic modulus to tensile strength, substantial strain hardening, a low-frequency viscoelastic dissipation of energy (e.g., as evidenced by the large hysteresis observed for a cyclic simple extension test), and substantial high-frequency inelastic dissipation of energy at the point of failure. The first three of these mechanisms can be used to explain the blunt crack tip and high-strain zones that form during a pure shear tear test (Figure 12). A small contribution is the viscoelastic deformation at high frequencies.
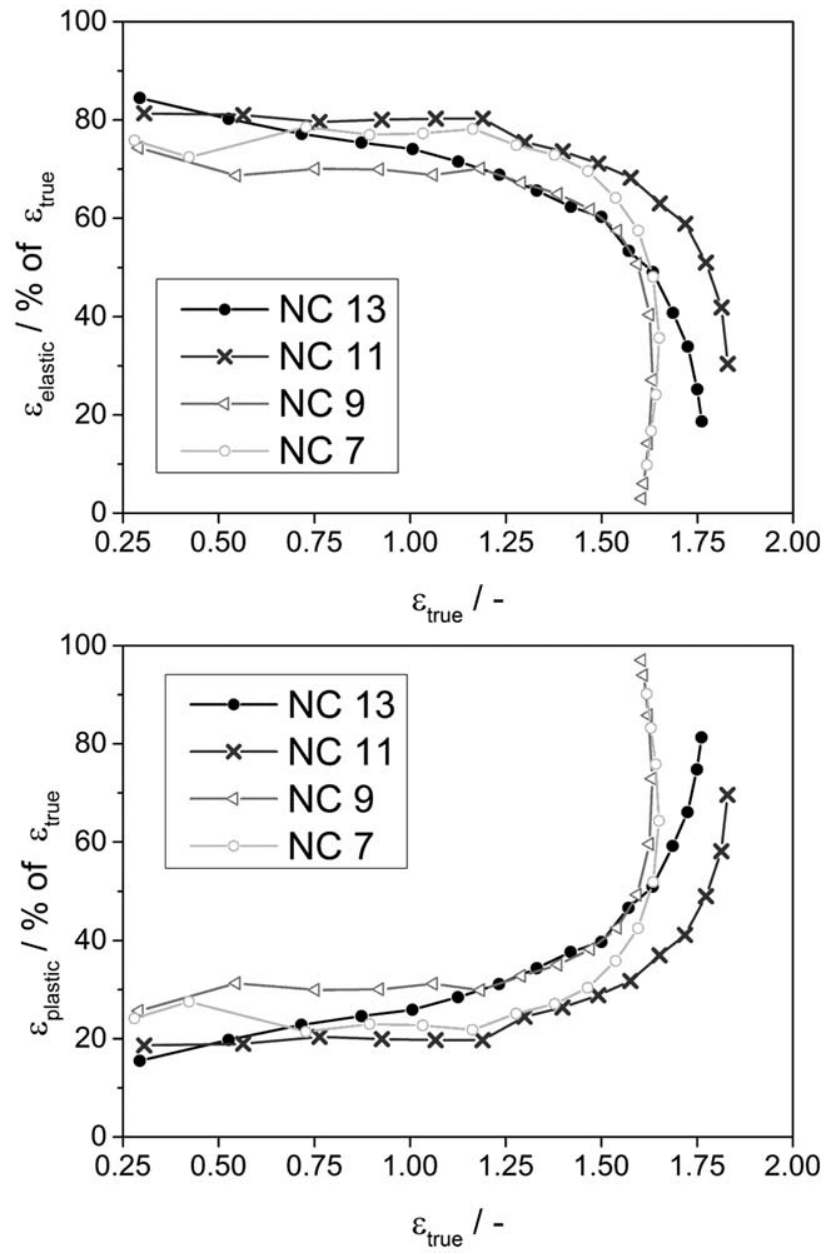

FIGURE 11 Top: Elastic true strain. Bottom: Plastic true strain, both in $\%$ of total true strain as a function of total true strain for samples NC7, NC9, NC11, and NC13. 


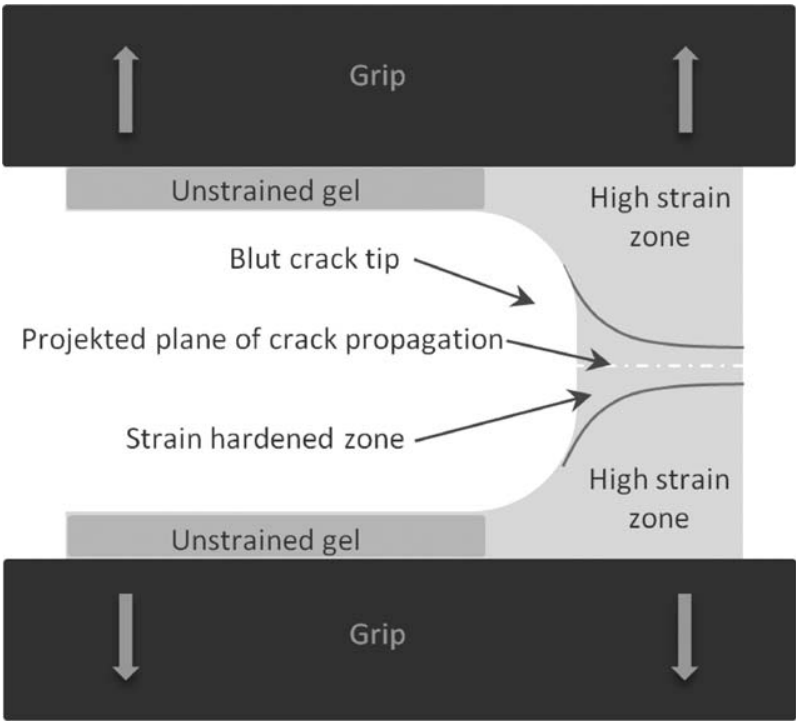

FIGURE 12 Schematic of the major deformation zones after applied strain but prior to failure in a pure shear tensile test of a PDMAA-NC hydrogel.

Because of the low ratio of elastic modulus to tensile strength, the PDMAA-NC hydrogels are able to blunt sharp crack tips at low applied strain via elastic deformation. As the stress concentration at the crack tip decreases as a crack tip radius increases, ${ }^{32}$ a blunt crack tip as present here implies that the stress concentration at the tip of a notch is minimized. Essential to attaining a low elastic modulus is a low density of polymer chains that corresponds to a large polymer molar mass between crosslinks. Any attempt to increase the elastic modulus that does not also increase the breaking strength by a similar portion will reduce the extent of elastic crack blunting and hence toughness. As such, the incorporation of Laponite XLS concentrations higher than $7.7 \mathrm{wt} \%$ (that used for NC13) or the incorporation of chemical crosslinks would reduce the extent of elastic crack blunting. It is pertinent to note that plastic deformation may also contribute to crack blunting at higher applied strains, but is unlikely to contribute at low strains.

Substantial strain hardening is crucial for the delocalization of strain. Without strain hardening, a relatively narrow band adjacent to the projected plane of crack growth would continue to stretch and fail without the formation of a high-strain zone extending to the grips. Strain hardening refers to an increasing tangent modulus as strain is increased. For natural rubber, strain hardening occurs due to strain-induced crystallization and is the key mechanism that stabilizes a balloon when it is inflated. For PDMAA, strain-induced crystallization is not feasible as the polymer is solvated. Furthermore, straininduced crystallization would create internal interfaces that would reflect light and hence change the hydrogel from transparent to opaque on stretching, which has not been observed to occur. It is well known that during stretching nanocomposite hydrogels, the Laponite crystal discs align parallel to the stretching direction. The alignment of the Laponite discs provides reinforcement and leads to strain hardening.
The high-strain zone shown in Figure 12 only forms because of strain hardening. Of high importance to toughness, only a small fraction of the work of extension can be released on time scales relevant to propagating a tear. The long-range elasticity of nanocomposite hydrogels is derived from the elasticity of polymer chains. However, the dissipation of energy responsible for the hysteresis is due to the formation and rupture of clay-clay and polymer-clay interactions. Clay-clay interactions are only significant at small strains and decrease as the clay discs are aligned during sample elongation. ${ }^{24,25}$ The rupture of polymer-clay interactions is anticipated to occur by segments of polymer chains peeling off individual Laponite discs. ${ }^{24,25}$ Lin et al. ${ }^{33}$ proposed that the formation and rupture of polymer-silica interactions enhanced the toughness of chemically crosslinked PDMAA-type hydrogels. Reports of self-healing nanocomposite hydrogels verify that polymer-clay interactions may rupture and form during deformation. ${ }^{17}$ Both the strength and density of these interactions determine the extent of hysteresis, and hence toughness. The sliding and rupture of PDMAA-Laponite interactions in the nanocomposite hydrogels is analogous to the polymer chains sliding over carbon black in carbon black-filled rubber. ${ }^{34}$

A tearing toughness as high as $6800 \mathrm{~J} \mathrm{~m}^{-2}$ is remarkably large, and there is a need to predict its magnitude to understand and synthesize better materials. One approach is to correlate the work of extension to the interaction energy and the number of polymer-clay interactions. ${ }^{35}$ By taking the tearing toughness fraction associated with elastic stored energy and using a Lake-Thomas approach, one may estimate the strength of polymer-clay interactions within the nanocomposite hydrogel.

The tear toughness values reported here refer to the measured toughness from a single extension. However, much of the work of extension is not instantaneously elastic as evidenced by the large hysteresis observed in the cyclic testing. The ratio of the work of extension for a single extension to the work of contraction for a strain of $500 \%$ was observed here to be $\sim 0.3$ (Fig. 10) and was reported by Tang et al. ${ }^{35}$ to be $\sim 0.07$ for a strain of $1500 \%$ for a PNIPA-NC (Laponite $\mathrm{XLG}$ ) hydrogel. By taking the work of extension to work of contraction ratios as upper and lower limits, one predicts a release of elastic energy release in the range of 476-2040 $\mathrm{J} \mathrm{m}^{-2}$ to propagate a crack for sample NC11. The lower limit of $476 \mathrm{~J} \mathrm{~m}^{-2}$ is much higher than that observed for a model polymer gel. ${ }^{10}$ Now, application of the Lake-Thomas model ${ }^{11}$ assumes that the tearing energy is equal to the energy required to dissociate a single polymer-clay interaction multiplied by the total number of polymer chain bonds between crosslinks that cross the plane of crack propagation:

$$
G_{0}=\left(\frac{3}{8}\right)^{1 / 2} C d^{*} U
$$

where $C$ is the concentration of DMAA at synthesis $\left(\mathrm{mol} \mathrm{m}^{-3}\right)$, $U$ is the polymer-clay interaction energy $\left(\mathrm{J} \mathrm{mol}^{-1}\right)$, and $d^{*}$ is the unstrained width of the damage zone $(\mathrm{m})$. By taking a polymer-clay interaction energy of $1.5 \mathrm{~kJ} \mathrm{~mol}^{-1}$, a value that is 
comparable with a representative hydrogen bond, ${ }^{35}$ one obtains an unstrained width of the damage zone of $0.34-$ $1.5 \mathrm{~mm}$. These widths are much larger than the contour length of the polymer chains, and it is clear that even at the point of failure, energy is being dissipated via polymer chains that do not intersect the fracture plane. DMA oscillation shear testing at frequencies between 0.01 and $10 \mathrm{~Hz}$ provided tangible evidence of viscoelastic energy dissipation that could occur at the time of failure.

One reported reason for the high tensile extensibility of nanocomposite gels is that the gels are free of microcracks and voids that typically occur in conventional organic gels during synthesis. ${ }^{36,37}$ Here, it was shown that nanocomposite gels are insensitive to notches that are orders of magnitude larger than the proposed microcracks and voids. By deduction, a model network structure free of microcracks and voids cannot by itself explain the insensitivity to notches, and hence cannot be a significant reason for the high toughness of nanocomposite hydrogels.

Probably the high fracture toughness of nanocomposite hydrogels is due to long chains between the crosslinking clay and the multifunctionality of this particular kind of crosslinker. If one polymer chain of one bond between polymer and clay is broken, nanocomposite hydrogels are capable of transferring its load to many other chains as a consequence of multifunctionality. ${ }^{31}$

\section{CONCLUSIONS}

Highly extendible nanocomposite hydrogels exhibit extraordinary toughness as high as $6800 \mathrm{~J} \mathrm{~m}^{-2}$ as determined by a single notch pure shear tear test. These gels are compliant and highly extendible. The nanocomposite gels were synthesized from DMAA and Laponite XLS, reagents, and a method similar to those in the literature. It is feasible that many of the reported nanocomposite hydrogels with similar stress-strain simple extension profiles should also exhibit high toughness.

The extraordinary toughness is attributed to both elastic and plastic crack blunting and a minor fraction of the work of extension contributing to crack growth. Elastic crack blunting is due to a large molar mass between effective crosslinks. A large hysteresis on tensile loading is an example of the minor work of extension that is restored on contraction and is attributed to the rupture and formation of clay-clay and polymer-clay interactions.

A combination of high-frequency DMA testing and lowfrequency stress relaxation and step cycle testing show that nanocomposite gels exhibit high viscoelastic loss of energy relative to conventional gels over time scales ranging from 0.1 up to 600 s. Step cycle testing show that the extent of viscoelastic loss will be reduced by cyclic tensile loading to strains higher than $425 \%$.

Nanocomposite hydrogels are insensitive to notches and are unlikely to exhibit brittle failure from tears and cuts.

\section{ACKNOWLEDGMENTS}

The authors acknowledge the use of facilities at the UOW Electron Microscopy Centre and the use of facilities at the University of Newcastle. Clay of type Laponite XLS®, a synthetic hectorite, was generously given by Rockwood Additives Ltd. (Princeton, NJ).

\section{REFERENCES AND NOTES}

1 K. Haraguchi, Macromol. Symp. 2007, 256, 120-130.

2 M. P. Linnes, B. D. Ratner, C. M. Giachelli, Biomaterials 2007, 28, 5298-5306.

3 P. Li, N. H. Kim, D. Hui, K. Y. Rhee, J. H. Lee, Appl. Clay Sci. 2009, 46, 414-417.

4 H. Kasgoz, A. Durmus, A. Kasgoz, I. Aydin, J. Macromol. Sci. Chem. 2012, 49, 92-99.

5 S. Hirotsu, Y. Hirokawa, T. Tanaka, J. Chem. Phys. 1987, 87, 1392.

6 G. Huang, J. Gao, Z. Hu, J. V. St. John, B. C. Ponder, D. Moro, J. Controlled Release 2004, 94, 303-311.

7 T. Hellweg, C. D. Dewhurst, E. Brückner, K. Kratz, W. Eimer, Colloid Polym. Sci. 2000, 278, 972-978.

8 C. He, Z. Zheng, D. Zhao, J. Liu, J. Ouyang, H. Wang, Soft Matter 2013, 9, 2837.

9 S. Naficy, H. R. Brown, J. M. Razal, G. M. Spinks, P. G. Whitten, Aust. J. Chem. 2011, 64, 1007.

10 Y. Akagi, H. Sakurai, J. P. Gong, U. Chung, T. Sakai, J. Chem. Phys. 2013, 139, 144905.

11 G. J. Lake, A. G. Thomas, Proc. R. Soc. A: Math. Phys. Eng. Sci. 1967, 300, 108-119.

12 J. P. Gong, Y. Katsuyama, T. Kurokawa, Y. Osada, Adv. Mater. 2003, 15, 1155-1158.

13 H. Xin, S. Z. Saricilar, H. R. Brown, P. G. Whitten, G. M. Spinks, Macromolecules 2013, 46, 6613-6620.

14 J. Y. Sun, X. Zhao, W. R. K. Illeperuma, O. Chaudhuri, K. H. Oh, D. J. Mooney, J. J. Vlassak, Z. Suo, Nature 2012, 489, 133-136.

15 K. Haraguchi, T. Takehisa, S. Fan, Macromolecules 2002, 35 , 10162-10171.

16 K. Haraguchi, T. Takehisa, Adv. Mater. 2002, 14, 1120.

17 G. Gao, G. Du, Y. Sun, J. Fu, ACS Appl. Mater. Interfaces 2015, 7, 5029-5037.

18 Okay, O, In Hydrogel Sensors and Actuators; G. Gerlach, K.-F. Arndt, Eds.; Springer: Berlin, Heidelberg, 2010; pp 1-14.

19 K. Haraguchi, H. J. Li, Macromolecules 2006, 39, 1898-1905.

20 K. Haraguchi, R. Farnworth, A. Ohbayashi, T. Takehisa, Macromolecules 2003, 36, 5732-5741.

21 BYK Additives and Instruments, Technical Information B-RI 21; LAPONITE: Performance Additives; BYK Additives and Instruments: Abelstraße, Germany. 2014.

22 K. Hong, A. Rastogi, G. Strobl, Macromolecules 2004, 37, 10165-10173.

23 R. S. Rivlin, A. G. Thomas, J. Polym. Sci. 1953, 10, 291-318.

24 T. Nishida, H. Endo, N. Osaka, H. J. Li, K. Haraguchi, M. Shibayama, Phys. Rev. E 2009, 80, 030801-1-030801-4.

25 T. Nishida, A. Obayashi, K. Haraguchi, M. Shibayama, Polymer 2012, 53, 4533-4538.

26 M. Mooney, J. Appl. Phys. 1940, 11, 582.

27 S. M. Gumbrell, L. Mullins, R. S. Rivlin, Trans. Faraday Soc. $1953,49,1495$ 
28 C. Y. Hui, A. Jagota, S. J. Bennison, J. D. Londono, Proc. $R$. Soc. A: Math. Phys. Eng. Sci. 2003, 459, 1489-1516.

29 V. Chalivendra, B. Song, D. Casem, In Proceedings of the 2012 Annual Conference on Experimental and Applied Mechanics; Springer: New York, NY, 2013, 125-133.

30 E. Evans, Annu. Rev. Biophys. Biomol. Struct. 2001, 30, 105128.

31 C. Creton, G. Josse, G. Miquelard, R. Webber, D. Hourdet, In Proceedings of the 13th International Conference on Deformation Yield and Fracture of Polymers; L. E. Govaert, H. E. H. Meijer, Eds.; Rolduc Abbey: Kerkrade, The Netherlands, 2006, 45-48.
32 T. L. Anderson, T. L. Anderson, Fracture Mechanics: Fundamentals and Applications, 3rd ed.; CRC Press: Hoboken, 2005. 33 W. C. Lin, W. Fan, A. Marcellan, D. Hourdet, C. Creton, Macromolecules 2010, 43, 2554-2563.

34 G. R. Hamed, Rubber Chem. Technol. 2000, 73, 524-533.

35 J. Tang, G. Xu, Y. Sun, Y. Pei, D. Fang, J Appl. Phys. 2014, 116, 244901.

36 T. Huang, H. G. Xu, K. X. Jiao, L. P. Zhu, H. R. Brown, H. L. Wang, Adv. Mater. 2007, 19, 1622-1626.

37 S. Miyazaki, T. Karino, H. Endo, K. Haraguchi, M. Shibayama, Macromolecules 2006, 39, 8112-8120. 